\section{Shroud irradiated with neutrons?}

Sir - If the shroud of Turin is in fact the burial cloth of Christ, contrary to its recent carbon-dated age of about 670 years (Nature 335, 663; 1988 and 337, 611; 1989), then according to the Bible it was present at a unique physical event: the resurrection of a dead body. Unfortunately, this event is not accessible to direct scientific scrutiny, but the image on the shroud, which still cannot be duplicated, appears to be a scorch, indicating that the body radiated light and/or heat. It may also have radiated neutrons, which would have irradiated the shroud and changed some of the nuclei to different isotopes by neutron capture. In particular, some ${ }^{14} \mathrm{C}$ could have been generated from ${ }^{13} \mathrm{C}$. If we assume that the shroud is 1,950 years old and that the neutrons were emitted thermally, then an integrated flux of $2 \times$ $10^{16}$ neutrons $\mathrm{cm}^{-2}$ would have converted enough ${ }^{13} \mathrm{C}$ to ${ }^{14} \mathrm{C}$ to give an apparent carbon-dated age of 670 years.

This flux of neutrons should have other measurable consequences. The neutron irradiation would probably not have been uniform, for example, so the ${ }^{14} \mathrm{C}^{13} \mathrm{C}$ ratio

should vary in different parts of the shroud. In addition, other unstable isotopes should have been formed. Several of these isotopes have half-lives long enough that they would still be present, yet short enough that they are not found naturally.

The unstable isotopes most likely to be found in the shroud are ${ }^{36} \mathrm{Cl}$ and ${ }^{41} \mathrm{Ca}$. The presence of either would confirm that the shroud had been irridated with neutrons An accurate measurement of the ratio of either ${ }^{36} \mathrm{Cl}$ to ${ }^{35} \mathrm{Cl}$ or ${ }^{+1} \mathrm{Ca}$ to ${ }^{41} \mathrm{Ca}$ (see table) would test the prediction of an integrated neutron flux of $2 \times 10^{16}$ neutrons $\mathrm{cm}^{-2}$ This may not be possible, however, because contamination with new sources of chlorine or calcium may have occurred from washings or other sources since the irridation took place.

Thomas J. Phillips

High Energy Physics Laboratory,

Harvard University,

Cambridge, Massachusetts 02138, USA

HEDGES REPLIES-The processes suggested by Phillips were considered by the participating laboratories. However, for the reasons given below, the likelihood

\begin{tabular}{cccccc}
\hline \multicolumn{5}{c}{ Properties of selected parent/daughter isotopes } \\
\hline $\begin{array}{c}\text { Parent } \\
\text { isotope }\end{array}$ & $\begin{array}{c}\text { Natural } \\
\text { abundance } \\
\text { (per cent) }\end{array}$ & $\begin{array}{c}\text { Neutron capture } \\
\text { cross-section } \\
\text { (barns) }\end{array}$ & $\begin{array}{c}\text { Daughter } \\
\text { isotope }\end{array}$ & $\begin{array}{c}\text { Half-life } \\
\text { (years) }\end{array}$ & $\begin{array}{c}\text { Predicted } \\
\text { parent/daughter } \\
\text { ratio }\end{array}$ \\
${ }^{13} \mathrm{C}$ & 1.11 & 0.0009 & ${ }^{14} \mathrm{C}$ & 5,730 & $1.2 \times 10^{-10}$ \\
${ }^{35} \mathrm{Cl}$ & 75.77 & 43 & ${ }^{36} \mathrm{Cl}$ & $3.0 \times 10^{5}$ & $8 \times 10^{-7}$ \\
${ }^{40} \mathrm{Ca}$ & 96.94 & 0.40 & ${ }^{44} \mathrm{Ca}$ & $1.0 \times 10^{5}$ & $8 \times 10^{-9}$
\end{tabular}

Predicted parent/daughter ratios (right-hand column) are for a new cloth irradiated with $2 \times 10^{16}$ neutrons $\mathrm{cm}^{-2}$. For ${ }^{14} \mathrm{C} /{ }^{13} \mathrm{C}$, the ratio is about 17 per cent higher than normal.

\section{Why reprints are necessary}

SiR-Ivor Smith (Nature 336, 708; 1988) complains of receiving reprint requests for a short paper and claims that "the cost of sending for this reprint and the time involved are appreciably greater than walking to the library and making a single photocopy for one's own use". He further suggests that photocopying articles, rather than sending reprint requests, is the "sensible approach".

Perhaps he is correct, if one reads only one short article each week. But in many scientific fields, including my own, one comes across 20-30 articles a week. The cost and time spent walking to the library, finding the articles and photocopying 30 articles is significantly greater than the cost and time required to fill out reprint requests. The volume of research articles is such that only with the time-efficient method of perusing Current Contents and requesting reprints of significant articles do I have a hope of having the time to actually read the articles. Thus, for many scientists, the reprints are requested on the basis of the title of the article, which is why it seems to Smith that the requesters have not yet read the article (and apparently will not read the article, considering that they do not receive the reprint).

If it is true that the "European reprint is a disappearing commodity", then perhaps the numbers of scientists reading European articles will be reduced, and European scientists themselves will be even more hard-pressed to keep up with the literature. This may be another funding-related factor which contributes to the decrease in scientific productivity in Britain, lamented in Nature over the past two years. Reprints may be expensive, but I believe that they remain the most time-efficient method of article acquisition, and will remain so until the development of 'electronic' journals.

Mathew T. Martin-Iverson

Neurochemical Research Unit,

Department of Psychiatry,

Mackenzie Health Sciences Centre,

University of Alberta,

Edmonton, Alberta,

Canada T6G 2B7 that they influenced the date in the way proposed is in my view so exceedingly remote that it beggars scientific credulity. (1) No plausible physical mechanism has been proposed to explain how the resurrection was accompanied by a significant neutron flux. If a supernatural explanation is to be proposed, it seems pointless to make any scientific measurement on the shroud at all.

(2) Assuming a 'scientific' (but not yet articulated) explanation for the neutron flux, it is an amazing coincidence that the neutron dose should be so exactly appropriate to give the most likely date on historical grounds. (Arguably a total of $10^{2 x}$ neutrons (the number in a human body) would be available. Using Phillips' figures, this would be sufficient to impart a date of 100,000 years into the future. To produce a date within 100 years of the first recorded history of the shroud implies that the dose has been 'fine-tuned' to better than one part in a hundred million.)

(3) In fact, the dose proposed by Phillips is much too high, as he has not included the neutron capture by nitrogen in the cloth. A not untypical $\mathrm{N}$ content in linen is 1,000 p.p.m., for which a thermal neutron flux of $2 \times 10^{13} \mathrm{~cm}^{-2}$ (that is, 1,000 times less) would be appropriate. This does not change the basic argument, but changes the chemical implications (even without recoil effects). The three dating laboratories used different types of chemical pretreatments, (for example at Oxford we purified the cellulose), yet obtained equivalent results. This shows that any ${ }^{14} \mathrm{C}$ formed by neutron irradiation behaves chemically in the same way as the original ${ }^{14} \mathrm{C}$. This is inherently unlikely because the original nitrogen is in a chemically quite different environment.

(4) As Phillips comments, one might expect that the 'irradiation' would be nonuniform. The three samples were contiguous, but at least on a local scale of 10 $\mathrm{mm}$, any such variation was less than 1 per cent.

The lower neutron flux would reduce the conversion of ${ }^{35} \mathrm{Cl}$ to ${ }^{36} \mathrm{Cl}$, but should still give a ratio significantly above background (if there has been no loss or gain of $\mathrm{Cl}$ since). Electron spin resonance signals from the 'irradiation' might also persist. However, such measurements are unlikely to confirm the presence or absence of neutron irradiation with absolute certainty. This, I fear, will not be achieved in a finite number of tests. If we accept a scientific result, we must exercise a critical notion of the probabilities involved. If we demand absolute certainty, we shall have to rely on faith.

Radiocarbon Accelerator Unit.

Research Laboratory for Archaeology and the History of Art,

University of Oxford,

6 Keble Road, Oxford OX2 $3 Q J, U K$ 